\section{Anatomical and functional retinal changes in multiple sclerosis}

G Cennamo1', MR Romano', EC Vecchio', C Minervino' ${ }^{1}$, C della Guardia ${ }^{1}$, N Velotti ${ }^{1}$, A Carotenuto², S Montella ${ }^{2}$, G Orefice ${ }^{2}$ and G Cennamo ${ }^{1}$
${ }^{1}$ Eye Clinic, Dipartimento di Neuroscienze e Scienze, Riproduttive ed Odontostomatologiche, Università degli Studi Federico II, Naples, Italy

${ }^{2}$ Neurological Clinic, Dipartimento di Neuroscienze e Scienze, Riproduttive ed Odontostomatologiche, Università degli Studi Federico II, Naples, Italy

Correspondence: MR Romano, Eye Clinic, Dipartimento di Neuroscienze e Scienze, Riproduttive ed Odontostomatologiche, Università degli Studi Federico II, Naples 80131, Italy

Tel: +39 0282244680;

Fax: +390282244980.

E-mail: mario.romano.md@ gmail.com

Received: 4 October 2014 Accepted in revised form: 31 October 2015

Published online:

18 December 2015

\begin{abstract}
Aims The aims of this study was to report anatomical changes of the ganglion cell complex (GCC), retinal nerve fiber layer (RNFL) thickness, and macular volume in patients with multiple sclerosis (MS). We also investigated the correlation between anatomical and functional changes in terms of visual acuity and macular sensitivity investigated and visual fields.

Methods Prospective comparative study included 105 eyes of 53 consecutive patients. The patients were divided into two groups: group A included 56 eyes of 28 patients with diagnosis of MS; group B involved 49 eyes of 25 healthy patients. The examination included Goldmann tonometry, biomicroscopic and fundus oculi examination, retinography, GCC examination, circumpapillary RNFL (cpRNFL), and macular volume. The functional test included measurement of best-corrected visual acuity (BCVA), visual field, and MP.

Results MS group showed a significant reduced GCC, cpRNFL, macular volume, BCVA, visual field, and macular sensitivity compared with the control group $(P<0.001)$. This reduction was more representative $(P<0.001)$ in patients with MS complicated by optic neuritis (ON). We found in the MS group a strong correlation between GCC thickness and macular volume $\left(r^{2}=0.59\right.$, $P<0.001)$ and also between GCC and RNFL thickness $\left(r^{2}=0.48, P<0.001\right)$. There was also a correlation between macular sensitivity and macular volume reduction $\left(r^{2}=0.25, P<0.001\right)$ and also between RNFL and macular volume $\left(r^{2}=0.43, P<0.001\right)$.

Conclusions The significant statistical evidence and the strong correlation between anatomical and functional parameters support the use of OCT and MP in the evaluation, treatment, and follow-up of patients diagnosed with MS.
\end{abstract}

Eye (2016) 30, 456-462; doi:10.1038/eye.2015.256; published online 18 December 2015

\section{Introduction}

Multiple sclerosis (MS) is a chronic, demyelinating, and inflammatory disease of the central nervous system (CNS) that mainly affect young adults. Along with the inflammatory processes, MS is characterized by a wide neurodegeneration, with axonal loss and neuronal rarefaction. ${ }^{1-6}$

Acute optic neuritis $(\mathrm{ON})$ is also a common feature of MS and it is the first clinical manifestation in $\sim 20 \%$ of patients affected by MS. ${ }^{1,2}$ Spectral-domain optical coherence tomography (OCT) offers a unique opportunity for sensitive monitoring of anatomical changes of axonal and neuronal loss. ${ }^{7,8}$ The objective of this study was to report anatomical changes of the ganglion cell complex (GCC), retinal nerve fiber layer (RNFL) thickness, and macular volume in patients with MS. We also investigated the correlation between SD-OCT structural changes and functional changes in terms of visual acuity and macular sensitivity, investigated with visual fields (VFs) and microperimetry (MP).

\section{Materials and methods}

\section{Study design}

Prospective comparative study included, from June 2012 to July 2013, 105 eyes of 53 consecutive patients. The study was performed in accordance with the ethical standards stated in the Declaration of Helsinki and approved by the institutional review board. The patients were followed in collaboration with the Eye clinic and Neurological Clinic, University of Naples 'Federico II'. Each patient signed an informed 
consent. The study has received no grant support from any agency.

\section{Participants}

The patients were divided into two groups: group A included 56 eyes of 28 patients, ranging from 19 to 58 years (mean 35.64, SD 10.68), with diagnosis of MS according to the McDonald criteria; ${ }^{9}$ group B involved 49 eyes of 25 healthy patients, ranging from 22 to 43 years (mean 35.98, SD 6.02). Between 25 healthy patients, 1 eye was excluded for previous local eye injuries. Table 1 describes demographical data of study patients. The eyes of group A were further divided into two groups: eyes with history of $\mathrm{ON}$ and eyes with no history of $\mathrm{ON}$. Twelve out of 56 eyes (21\%) in group A presented with a history of ON. A history of ON was recorded in the presence of an acute relapse lasting more than $24 \mathrm{~h}$ and associated with decreased high-contrast letter acuity, pain with eye movement, color desaturation, and/or VF abnormalities, with or without optic nerve swelling or enhancement visualized by magnetic resonance imaging. The exclusion criteria were the presence of congenital eye diseases, previous episodes of ON occurred in the 3 months before the examination, previous episodes of inflammation of the optic nerve because of other diseases, tobacco and/or addiction alcoholism, glaucomatous optic neuropathy, ocular media opacities, high myopia, and pre-existing macular disease. All controls, group B, had a best-corrected visual acuity (BCVA) equal to 20/20 and a refractive spherical equivalent error between +2.00 and -2.00 diopters. No significant differences were found in age between patients and the control group. All patients and all healthy controls underwent a collection of personal and family history, a complete neurological examination with evaluation of EDSS (Expanded Disability Status Scale), and an eye examination

The eye examination included Goldmann tonometry (AT900; Haag-Streit, Koeniz, Switzerland), biomicroscopic and fundus oculi examination, retinography, GCC examination, circumpapillary RNFL

Table 1 Characteristics of the study population

\begin{tabular}{lcccc}
\hline & \multicolumn{2}{c}{ Group A } & & Group B \\
\cline { 2 - 3 } & $\begin{array}{c}\text { Multiple } \\
\text { sclerosis with } \\
\text { ON }\end{array}$ & $\begin{array}{c}\text { Multiple } \\
\text { sclerosis without ON }\end{array}$ & Control \\
& 12 & 16 & \\
\hline Participants (no.) & 12 & 32 & & 49 \\
Eyes (no.) & 24 & $35.64 \pm 10.68$ & & $34.98 \pm 6.02$ \\
Age (years) & $32.90 \pm 9.75$ & $9 / 7$ & & $15 / 14$ \\
Male/female & $2 / 10$ & $2.18 \pm 1.23$ & - \\
Mean EDSS & $2.86 \pm 1.82$ & & - \\
IOP (mm Hg) & $16.18 \pm 2.35$ & $15.42 \pm 2.64$ & $15.58 \pm 2.39$ \\
\hline
\end{tabular}

(cpRNFL), and macular volume. The functional test included measurement of visual BCVA, VF, and MP. The pharmacological mydriasis (minimum $5 \mathrm{~mm}$ pupil diameter) was obtained with one drop of tropicamide $1 \%$ and one drop of phenylephrine $2.5 \%$ at least $15 \mathrm{~min}$ before the SD-OCT and MP examination. For each patient, all exams were performed in a single day and by two operators.

\section{Examination}

Visual acuity We measured according to the Early Treatment Diabetic Retinopathy Study chart, which was converted to a logarithm of the minimum angle of resolution $\left(\log\right.$ MAR) for statistical analysis. ${ }^{10}$

SD-OCT GCC and cpRNFL thickness were evaluated by RTVue-100 OCT (Optovue Inc., Fremont, CA, USA; software version 4.0.5.39). RTVue-100 OCT acquires 26000 axial scans (a-scans) per second and has a 5 - $\mu \mathrm{m}$-depth resolution (full-width half-maximum) in tissue. The optic nerve head map protocol was applied to evaluate the cpRNFL. This protocol generates a cpRNFL thickness map based on measurements obtained along a circle $3.45 \mathrm{~mm}$ in diameter centered on the optic disc. The GCC protocol was used to determine the GCC and macular thickness. Image quality was carefully checked after each acquisition: images were not used when the signal strength was $<40$, there was the presence of a misalignment of the surface detection algorithm, or there was an obvious decentration of the measurement circle.

The fundus was well illuminated, the ring scan was correctly centered, and the measurement beam was placed centrally; red lines for detection algorithm correctly identified the superior and inferior RNFL border for ring scans and the retinal borders for volume scans. ${ }^{11}$

Pharmacological dilatation was performed in all subjects to ensure optimal image quality and to permit careful retinal segmentation. The photographers rejected scans with motion artifacts (discontinuous jump). The GCC thickness was measured from the internal limiting membrane to the outer boundary of the inner plexiform layer. Controls were obtained averaging, over seven examinations, both the mean values of GCC thickness and of cpRNFL in the contralateral eyes. The ratio of the affected eye measurement to the contralateral eye measurement was evaluated at each session.

Microperimetry Fundus-related microperimetry (MP-1; Microperimeter Nidek Technologies, Padua, Italy) was performed on all eyes using the NAVIS software version 1.7.2 (Microperimeter Nidek Technologies). ${ }^{8,9,11-13}$ Briefly, we used the following setting in this study: a fixation target consisting of a red ring $2^{\circ}$ in diameter; white, 
monochromatic background at $4 \mathrm{asb}$, stimulus size Goldman III with 200 ms projection time; customized radial grid of 61 stimuli covering the central $10^{\circ}$ (centered on the fovea); stimuli were projected randomly onto the $0^{\circ}, 30^{\circ}, 60^{\circ}, 90^{\circ}, 120^{\circ}$, and $150^{\circ}$ axes, $1^{\circ}$ apart. A $4-2-1$ double staircase strategy was used with an automatic eye tracker that compensates for eye movements. To evaluate the clinical correspondence between microperimetric data and retinal details, functional results were displayed onto a color digital retinograph, acquired by the charge-coupled device color camera of the microperimeter.

Visual field The VF examination was performed with a computerized automatic static perimeter (HFA; Swedish Interactive Thresholding Algorithm (SITA) standard; Carl Zeiss Meditec Inc., Dublin, CA, USA). The luminance of the background is 31.5 asb with a Stimulus III white/ white. The threshold strategy examines $24^{\circ}$ field of view on 54 points (test SITA standard 24-2). ${ }^{14}$ The points are spaced from each other by $6^{\circ}$. The $\mathrm{VF}$ is considered reliable when fixation losses were $<20 \%$, and falsepositive and false-negative errors $<15 \%$. The average sensitivity was calculated by the software and expressed as the MD (mean deviation) and PSD (pattern standard deviation).

Statistical analyses Statistical analysis was performed using the Statistical Package for Social Sciences (version 15.0 for Windows; SPSS Inc., Chicago, IL, USA). The twotailed unpaired $T$-test was used to evaluate differences between controls and MS and between MS eyes with and without ON. The considered parameters were mean GCC thickness, global loss volume (GLV) and focal loss volume (FLV), cpRNFL, macular volume, macular sensitivity, and MD-HVF, PSD-HVF.

Furthermore, in the control group and MS group, Pearson's correlation coefficients ( $r$ values), linear regression, and determination coefficient $\left(r^{2}\right)$ were calculated between BCVA (logMAR) and mean GCC thickness; BCVA (logMAR) and macular sensitivity between macular sensitivity and macular volume and also between RNFL and macular volume. A $P$-value of $<0.05$ was considered statistically significant.

\section{Results}

Twenty-eight patients (17 females, 11 males, mean age $35.64 \pm 10.68$ years) with 56 total eyes examined were included in the study. Twelve eyes of MS patients had ON.

EDSS disability score was 2 (range $0-4$ ). The control group was composed of 25 patients ( 14 females, 11 males, mean age $34.98 \pm 6.02$ years) with 49 total eyes. The characteristics of both groups are summarized in Table 1 .

\section{Anatomical outcomes}

In the MS group, GCC thickness was significantly reduced comparing with control eyes $(86.73 \pm 10.25$ vs $98.89 \pm 4.65 \mu \mathrm{m}, P$-value $<0.001)$; this reduction was more representative in ON eyes $(79.9 \pm 9.68$ vs $98.89 \pm 4.65 \mu \mathrm{m}$, $P$-value $<0.001)$. Also, other parameters considered, FLV and GLV, had significant reduction in the MS group $(P$-value $<0.001)$ (Table 2$)$. In the MS group, the cpRNFL was reduced when compared with the control group $(94.86 \pm 11.64$ vs $109.77 \pm 19.54 \mu \mathrm{m}, P$-value $<0.001)$. Once again, the reduction was more representative in ON eyes $(87.84 \pm 10.95 \mu \mathrm{m}$ vs $109.77 \pm 19.54 \mu \mathrm{m}$, $P$-value $<0.001)$ (Table 2). Furthermore, in the MS group, macular volume was reduced in study patients $\left(6.98 \pm 0.34\right.$ vs $7.22 \pm 0.23 \mathrm{~mm}^{3}, P$-value $\left.=0.005\right)$. In $\mathrm{ON}$ eyes, macular volume was even thinner than the control group $\left(6.76 \pm 0.21\right.$ vs $7.22 \pm 0.23 \mathrm{~mm}^{3}, P$-value $\left.<0.005\right)$.

Finally, comparing eyes with the absence or presence of $\mathrm{ON}$, there were a significant statistical difference in GCC thickness $(86.73 \pm 10.25$ vs $79.9 \pm 9.68 \mu \mathrm{m}, P$-value $<0.05)$,

Table 2 GCC thickness, cpRNFL thickness, MV, MS and automated perimetry ${ }^{\mathrm{a}}$

\begin{tabular}{|c|c|c|c|c|c|}
\hline & $\begin{array}{c}\text { Control } \\
(\text { mean } \pm S D)\end{array}$ & $\begin{array}{c}\text { MS without } \\
\text { ON (mean } \pm S D)\end{array}$ & $\begin{array}{c}M S \text { with } O N \\
(\text { mean } \pm S D)\end{array}$ & $\begin{array}{l}\text { P-value MS } \\
\text { without ON }\end{array}$ & $\begin{array}{c}\text { P-value } \\
\text { MS with ON }\end{array}$ \\
\hline BCVA (log MAR) & $0 \pm 0$ & $0.05 \pm 0.10$ & $0.08 \pm 0.16$ & $<0.001$ & $<0.001$ \\
\hline Avg GCC $(\mu \mathrm{m})$ & $98.89 \pm 4.65$ & $86.73 \pm 10.25$ & $79.9 \pm 9.68$ & $<0.001$ & $<0.001$ \\
\hline FLV (\%) & $0.41 \pm 0.42$ & $2.99 \pm 4.05$ & $5.96 \pm 3.56$ & $<0.001$ & $<0.001$ \\
\hline GLV $(\%)$ & $3.02 \pm 2.08$ & $12.28 \pm 8.98$ & $18.8 \pm 7.41$ & $<0.001$ & $<0.001$ \\
\hline Macular volume $\left(\mathrm{mm}^{3}\right)$ & $7.22 \pm 0.23$ & $6.98 \pm 0.34$ & $6.76 \pm 0.21$ & 0.005 & $<0.005$ \\
\hline RNFL average $(\mu \mathrm{m})$ & $109.77 \pm 19.54$ & $94.86 \pm 11.64$ & $87.84 \pm 10.95$ & $<0.001$ & $<0.001$ \\
\hline Macular sensitivity $(\mathrm{dB})$ & $19.49 \pm 0.31$ & $17.17 \pm 2.24$ & $16.55 \pm 2.04$ & $<0.001$ & $<0.001$ \\
\hline MD-HVF (dB) & $0.08 \pm 1.13$ & $-1.32 \pm 2.44$ & $-2.26 \pm 2.86$ & 0.004 & $<0.005$ \\
\hline PSD-HVF (dB) & $1.42 \pm 0.6$ & $2.69 \pm 1.72$ & $2.88 \pm 1.69$ & $<0.001$ & $<0.001$ \\
\hline
\end{tabular}

Abbreviations: cpRNFL, circumpapillary retinal nerve fiber layer; GCC, ganglion cell complex; MS, macular sensitivity; MV, macular volume; ON, optic neuritis. ${ }^{\text {In }}$ the control group, eyes vs eyes with MS without optic neuritis and vs eyes with MS with ON. 
FLV $(2.99 \pm 4.05$ vs $5.96 \pm 3.56 \mu \mathrm{m}, P$-value $<0.05)$, GLV $(12.28 \pm 8.98$ vs $18.8 \pm 7.41 \mu \mathrm{m}, P$-value $<0.05)$. (Table 3$)$.

\section{Functional outcomes}

In MS group, the mean BCVA was $0.05 \pm 0.10 \log$ MAR and IOP was $15.27 \pm 2.24 \mathrm{~mm} \mathrm{Hg}$, whereas the MS-ON group presented mean BCVA of $0.08 \pm 0.16 \log \mathrm{MAR}$ and a IOP of $16.18 \pm 2.35 \mathrm{~mm} \mathrm{Hg}$.
Macular sensitivity was statistically significantly reduced in MS patients when compared with the control group $(17.17 \pm 2.24$ vs $19.49 \pm 0.31 \mathrm{~dB}, P$-value $<0.001)$. This reduction was more representative in $\mathrm{ON}$ eyes $(16.55 \pm 2.04$ vs $19.49 \pm 0.31 \mathrm{~dB}, P$-value $<0.001)$. We also found central fixation in 49 eyes $(87.50 \%)$ and eccentric fixation in 4 eyes $(7.14 \%)$ with ON. (Figure 1).

Compared with the control group, the automated perimetry in the MS group revealed a reduction both in MD $(-1.32 \pm 2.44$ vs $0.08 \pm 1.13 \mathrm{~dB}, P$-value $=0.004)$

Table 3 GCC thickness, cpRNFL thickness, MV, MS, and automated perimetry ${ }^{\mathrm{a}}$

\begin{tabular}{lccc}
\hline & MS without ON (mean \pm SD) & MS with ON (mean \pm SD) & P-value \\
\hline BCVA (logMAR) & $0.05 \pm 0.10$ & $0.08 \pm 0.16$ & $>0.05$ \\
Avg GCC $(\mu \mathrm{m})$ & $86.73 \pm 10.25$ & $79.9 \pm 9.68$ & $<0.05$ \\
FLV $(\%)$ & $2.99 \pm 4.05$ & $5.96 \pm 3.56$ & $<0.05$ \\
GLV $(\%)$ & $12.28 \pm 8.98$ & $18.8 \pm 7.41$ & $<0.05$ \\
Macular volume $\left(\mathrm{mm}^{3}\right)$ & $6.98 \pm 0.34$ & $6.76 \pm 0.21$ & $<0.05$ \\
RNFL average $(\mu \mathrm{m})$ & $94.86 \pm 11.64$ & $87.84 \pm 10.95$ & 0.066 \\
Macular sensitivity $(\mathrm{dB})$ & $17.17 \pm 2.24$ & $16.55 \pm 2.04$ & $>0.05$ \\
MD-HVF $(\mathrm{dB})$ & $-1.32 \pm 2.44$ & $-2.26 \pm 2.86$ & $>0.05$ \\
PSD-HVF $(\mathrm{dB})$ & $2.69 \pm 1.72$ & $2.88 \pm 1.69$ & $>0.05$ \\
\hline
\end{tabular}

Abbreviations: cpRNFL, circumpapillary retinal nerve fiber layer; GCC, ganglion cell complex; MS, macular sensitivity; MV, macular volume; ON, optic neuritis. ${ }^{a}$ In eyes with and without ON.

a
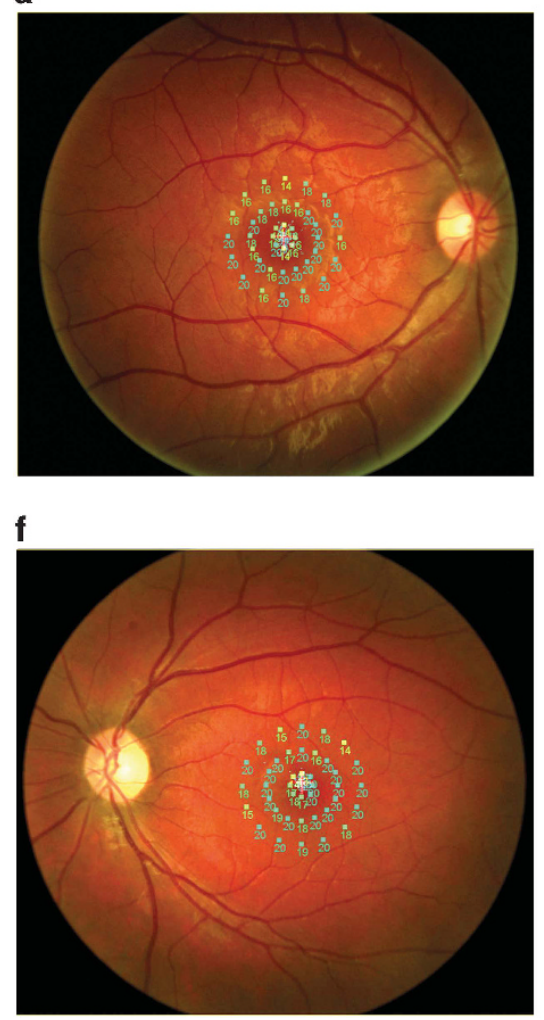

b

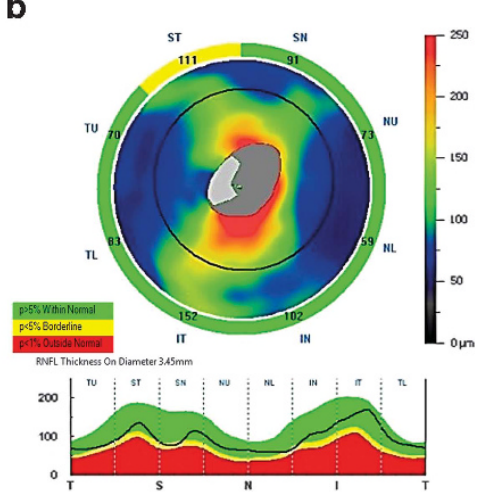

g

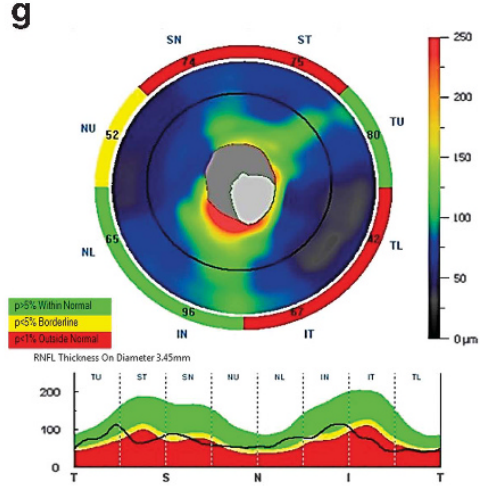

c

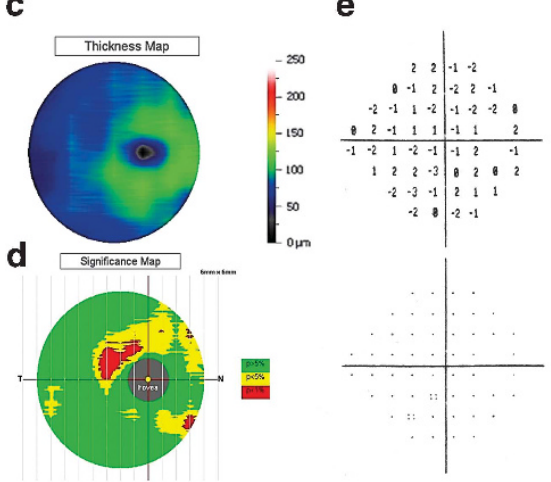

h

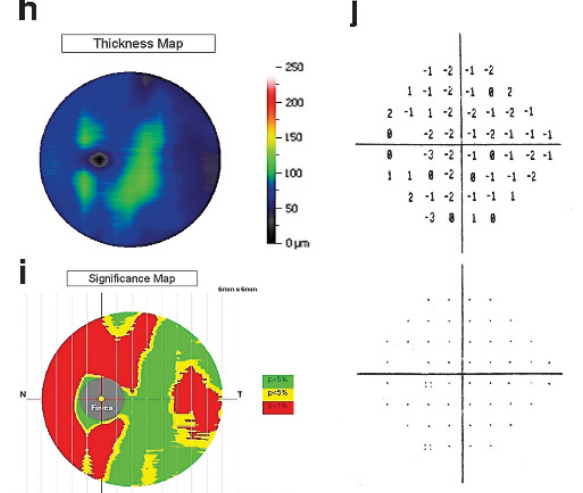

Figure 1 The figure shows an initial form of MS in patient with a slight decrease of macular sensitivity (a) associated with a decrease of the RNF layer (b), GCC (c and d) thickness, and VF (e). The figure also show another patient affected by MS with a slight decrease of macular sensitivity (f) associated with a significant decrease of RNFL (g) and GCC (h and j) thickness and slight changes in VF (i). 
and PSD $(2.69 \pm 1.72$ vs $1.42 \pm 0.6 \mathrm{~dB}, P$-value $<0.001)$; in the MS-ON group, all eyes had a more significant MD reduction $(-2.26 \pm 2.86$ vs $0.08 \pm 1.13 \mathrm{~dB}$,

$P$-value $<0.005)$.

Furthermore, eyes with ON did not show a significant statistical reduction of functional parameters when compared with eyes without ON.

\section{Correlations}

Finally, we found in the MS group a strong correlation between GCC thickness and macular volume $\left(r^{2}=0.59\right.$, $P<0.001)$ and between GCC and RNFL thickness $\left(r^{2}=0.48, P<0.001\right)$ (Figure 2).

There was also a correlation between macular sensitivity and macular volume reduction $\left(r^{2}=0.25\right.$, $P<0.001)$ and also between RNFL and macular volume $\left(r^{2}=0.43, P<0.001\right)$.

\section{Discussion}

Our study evaluated both anatomic and functional features in patients with MS using SD-OCT and MP, looking closely to the correlation between $\mathrm{ON}$ damage and macular changes. In the previous study, Shindler et $a l^{1,2}$ demonstrated the progressive apoptosis of retinal ganglion cells after an acute episode of $\mathrm{ON}$ in murine models. ${ }^{1,2}$ On the other hand, in 2011, Quinn et al ${ }^{3}$ demonstrated the apoptosis of retinal ganglion cells also in the absence of acute inflammation of optic nerve after induction of autoimmune encephalomyelitis in the murine model; the authors suggested that it could be leaked to a specific genetic pattern or to the oxidative stress.

In 2010, Green et al ${ }^{4}$ demonstrated the reduction both of GCC and inner nuclear layer (INL) thickness in 82 patients with MS; they also found the complete absence of inflammation cells in the INL and supposed it was related to the effect of a retrograde transynaptic degeneration. The authors also proposed the presence of a not-identified virus, which could determinate an autoimmune response. ${ }^{4}$ In 2013, Kaushik et al ${ }^{5}$ demonstrated the relationship between retinal ganglion cell loss and changes of INL in ON, demonstrating that INL enlargement in MS-related ON is associated with the severity of GCC loss. It has also been reported that the RNFL thickness and the macular volume with OCT is helpful to investigate the real axonal damage because this measurement is not altered by the process of demyelination, which MS patients are involved in. ${ }^{6}$

In 1999, Parisi et $\mathrm{al}^{7}$ demonstrated a reduction of RNFL thickness in patients with MS and acute episodes of ON. Costello et $a l^{8}$ in 2006 suggested that this reduction of RNFL thickness is about $10-40 \mu \mathrm{m}$ in 6 months after ON. Other authors confirmed that the axonal loss, evaluated as RNFL thickness reduction, is related with visual dysfunction and could be used as a global measurement of disability in patients with MS. ${ }^{12-16}$ Pulicken et al ${ }^{17}$ in 2007 demonstrated that the RNFL thickness was more reduced in patients with secondary progressive MS than in relapsing-remitting MS; this suggested that RNFL thickness could represent a marker of progression of the disease.

In 2005, Trip et al ${ }^{18}$ verified the correlation between RNFL thickness reduction and macular volume reduction in eyes with and without ON; they suggested that in MS there is a retrograde axonal degeneration with a loss of retinal ganglion cells and a consequent macular volume reduction.

In 2012, Zimmerman et al ${ }^{19}$ investigated the association of white and grey matter brain volume with peripapillary RNFL and GCC in MS patients with and without a history of $\mathrm{ON}$, demonstrating that both parameters of neuroaxonal damage are comparably linked to whole brain as well as white and grey matter atrophy. At same
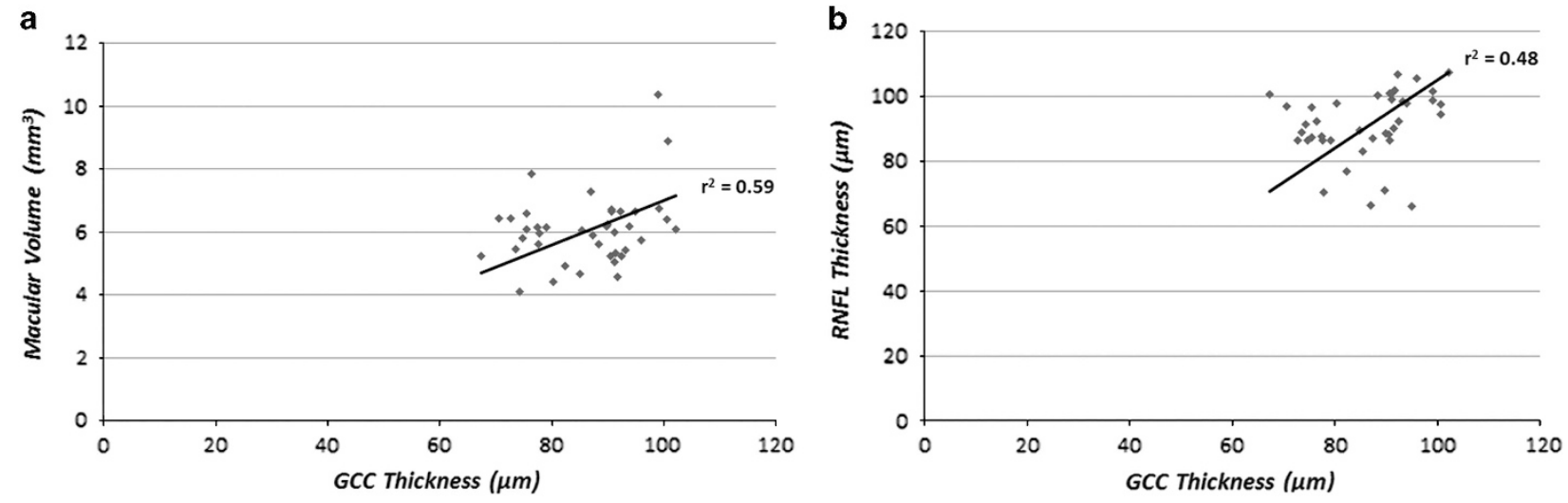

Figure 2 The figure shows the regression line of correlation between GCC thickness and macular volume in the MS group (a). The figure (b) also shows the regression line of correlation between GCC and RNFL thickness in the MS group. 
time, Tátrai et $a l^{20}$ evaluated the macular morphology in MS patients with OCT; they found a reduction of RNFL, GCC, and inner plexiform layer thickness in eyes of MS patients regardless of previous $\mathrm{ON}$, underlining the important role in the diagnosis and follow-up of this pathology. By this point of view, the modern FD-OCT, owing to computerized retinal segmentation algorithms, will continue to facilitate the detection of GCC and macular volume as demonstrated in many recent studies. $^{21,22}$

In 2007, Romano et al ${ }^{23}$ documented a loss of sensitivity in the macular region of the eye of MS patient with ON, demonstrating that the MP could be an interesting tool to evaluate the macular and peripapillary region. The author reports the efficacy of MP-1 in detecting early neuronal damage as the changes in macular sensitivity, even before BCVA decreases. Furthermore, the association of fundus photography and tracking system allows us to focus on the macula area to test the exact same points at each follow-up, as an useful tool to study the subclinical form of $\mathrm{ON}$ monitor the systemic treatment of MS. ${ }^{23}$

In the current study, we documented a significant reduction of GCC thickness, RNFL thickness, and macular volume when compared with the control group. We also found a significant reduction of macular sensibility in patients with MS and ON. Finally, our results demonstrate a strong correlation between macular sensibility and macular volume and also between macular sensibility and GCC and RNFL thickness. We also found a stronger reduction of GCC thickness in patients with eccentric fixation (GCC average $<80 \mu \mathrm{m}$ ). We believe that our significant statistical evidence and the strong correlation between anatomical and functional parameters support the use of OCT and MP in clinical trial measures of patients diagnosed with MS.

\section{Summary}

What was known before

- MS induces anatomical and functional changes of the GCC retinal nerve fiber thickness. We report statistical evidence and strong correlation between anatomical and functional parameters, supporting the use of OCT and MP in the evaluation, treatment, and follow-up of patients diagnosed with MS.

\section{What this study adds}

- Statistical evidence and strong correlation between anatomical and functional changes detected with OCT and MP in patients affected by MS.

\section{Conflict of interest}

The authors declare no conflict of interest.

\section{References}

1 Shindler KS, Guan Y, Ventura E, Bennett J, Rostami A. Retinal ganglion cell loss induced by acute optic neuritis in a relapsing model of multiple sclerosis. Mult Scler 2006; 12: 526-532.

2 Shindler KS, Ventura E, Dutt M, Rostami A. Inflammatory demyelination induces axonal injury and retinal ganglion cell apoptosis in experimental optic neuritis. Exp Eye Res 2008; 87: 208-213.

3 Quinn TA, Dutt M, Shindler KS. Optic neuritis and retinal ganglion cell loss in a chronic murine model of multiple sclerosis. Front Neurol 2011; 2: 50.

4 Green AJ, McQuaid S, Hauser SL, Allen IV, Lyness R. Ocular pathology in multiple sclerosis: retinal atrophy and inflammation irrespective of disease duration. Brain 2010; 133: 1591-1601.

5 Kaushik M, Wang CY, Barnett MH, Garrick R, Parratt J, Graham SL et al. Inner nuclear layer thickening is inversley proportional to retinal ganglion cell loss in optic neuritis. PLoS One 2013; 8: e78341.

6 Henderson AP, Trip SA, Schlottmann PG, Altmann DR, Garway-Heath DF, Plant GT et al. A preliminary longitudinal study of the retinal nerve fiber layer in progressive multiple sclerosis. J Neurol 2010; 257: 1083-1091.

7 Parisi V, Manni G, Spadaro M, Colacino G, Restuccia R, Marchi S et al. Correlation between morphological and functional retinal impairment in multiple sclerosis patients. Invest Ophthalmol Vis Sci 1999; 40: 2520-2527.

8 Costello F, Coupland S, Hodge W, Lorello GR, Koroluk J, Pan YI et al. Quantifying axonal loss after optic neuritis with optical coherence tomography. Ann Neurol 2006; 59: 963-969.

9 Polman CH, Reingold SC, Banwell B, Clanet M, Cohen JA, Filippi M et al. Diagnostic criteria for multiple sclerosis: 2010 revisions to the McDonald criteria. Ann Neurol 2011; 69: 292-302.

10 Kaiser PK. Prospective evaluation of visual acuity assessment: a comparison of snellen versus ETDRS charts in clinical practice. Trans Am Ophthalmol Soc 2009; 107: 311-324.

11 Tewarie P, Balk L, Costello F, Green A, Martin R, Schippling S et al. The OSCAR-IB consensus criteria for retinal OCT quality assessment. PLoS One 2012; 7 (4): e34823.

12 Costello F, Hodge W, Pan YI, Freedman M, De Meulemeester C. Differences in retinal nerve fiber layer atrophy between multiple sclerosis subtypes. J Neurol Sci 2009; 281: 74-79.

13 Cheng H, Laron M, Schiffman JS, Tang RA, Frishman LJ. The relationship between visual field and retinal nerve fiber layer measurement in patients with multiple sclerosis. Invest Ophthalmol Vis Sci 2007; 48: 5798-5805.

14 Rebolleda G, García-García A, Won Kim HR, Muñoz-Negrete FJ. Comparison of retinal nerve fiber layer measured by time domain and spectral domain optical coherence tomography in optic neuritis. Eye (Lond) 2011; 25: 233-238.

15 Gordon-Lipkin E, Chodkowski B, Reich DS, Smith SA, Pulicken M, Balcer LJ et al. Retinal nerve fiber layer is associated with brain atrophy in multiple sclerosis. Neurology 2007; 69: 1603-1609.

16 Toledo J, Sepulcre J, Salinas-Alaman A, Garcia-Layana A, Murie-Fernandez M, Bejarano B et al. Retinal nerve fiber layer atrophy is associated with physical and cognitive disability in multiple sclerosis. Mult Scler 2008; 14: 906-912. 
17 Pulicken M, Gordon-Lipkin E, Balcer LJ, Frohman E, Cutter G, Calabresi PA. Optical coherence tomography and disease subtype in multiple sclerosis. Neurology 2007; 69: 2085-2092.

18 Trip SA, Schlottmann PG, Jones SJ, Altmann DR, GarwayHeath DF, Thompson AJ et al. Retinal nerve fiber layer axonal loss and visual dysfunction in optic neuritis. Ann Neurol 2005; 58: 383-391.

19 Zimmermann H, Freing A, Kaufhold F, Gaede G, Bohn E, Bock $\mathrm{M}$ et al. Optic neuritis interferes with optical coherence tomography and magnetic resonance imaging correlations. Mult Scler 2013; 19: 443-450.

20 Tátrai E, Simó M, Iljicsov A, Németh J, Debuc DC, Somfai GM. In vivo evaluation of retinal neurodegeneration in patients with multiple sclerosis. PLoS One 2012; 7: e30922.
21 Watson GM, Keltner JL, Chin EK, Harvey D, Nguyen A, Park SS. Comparison of retinal nerve fiber layer and central macular thickness measurements among five different optical coherence tomography instruments in patients with multiple sclerosis and optic neuritis. J Neuroophthalmol 2011; 31: $110-116$.

22 Davydovskaia MV, Tsysar MA, Boiko AN, Akopian VS, Semenova NS, Filonenko IV et al. Damage of macular ganglion cell complex and peripapillary retinal nerve fiber layer in multiple sclerosis. Zh Nevrol Psikhiatr Im S S Korsakova 2012; 112: 47-51.

23 Romano MR, Angi M, Romano F. Macular sensitivity change in multiple sclerosis followed with microperimetry. Eur J Ophthalmol 2007; 17: 441-444. 